Available online at:
Thtp://ejournal.amikompurwokerto.ac.id/index.php/telematikal
Telematikatika
Accreditated SINTA “2” Kemenristek/BRIN, No. 85/M/KPT/2020

\title{
CNN Architecture for Classifying Types of Mango Based on Leaf Images
}

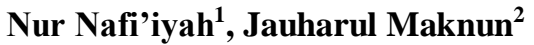 \\ ${ }^{1}$ Lecturer Informatics Engineering, Engineering \\ ${ }^{2}$ Student Informatics Engineering, Engineering \\ Universitas Islam Lamongan \\ E-mail: mynaff@unisla.ac.id ${ }^{1}$, jauharulmaknun001@ gmail.com²
}

\begin{tabular}{l}
\hline A R T I C L E I N F O \\
\hline History of the article: \\
Received April 9, 2021 \\
Revised June 6, 2021 \\
Accepted August 16, 2021 \\
Available online August 31, \\
2021 \\
\\
\hline Keywords: \\
Mango leaves \\
classification \\
transfer learning \\
CNN architecture \\
Correspondece: \\
Telepon: +62 (0751) 12345678 \\
E-mail: mynaff26@gmail.com
\end{tabular}

\section{INTRODUCTION}

Processing the digital image, vision techniques computer, machine learning algorithms, and deep learning are increasingly being developed due to handling complex data and good precision results (Chouhan et al., 2019). The stages in developing a computer-based automation system (Yamparala et al., 2020) based on images need to be processed and segmented images (Razi, 2012), then learning to find out the pattern of an image (Chouhan et al., 2019) (Prasad et al., 2019) al., 2016). The goal of developing an automation system in agriculture is to help the agricultural team, as well as maximum agrarian output, and facilitate more efficient work (Yamparala et al., 2020) (Ranjan et al., 2015) (Arivazhagan et al., 2013) ( Samajpati \& Degadwala, 2016) (Mishra et al., 2021) (Zarrin \& Islam, 2019). Not everyone knows the ins and outs of agriculture because not everyone studies in that field. Therefore, people in agriculture are trying to develop a system that can classify types of plants, identify plant diseases. The hope is that they can help others who do not study in agriculture to take good care of plants and help facilitate the work of caring for plants (Yamparala et al., 2020) (Aakif \& Khan, 2015) (Prasetyo, 2016) (Arivazhagan et al., 2013) ) (Samajpati \& Degadwala, 2016) (Mishra et al., 2021). Farmers who work in the agricultural sector also 
sometimes have difficulty identifying the type of plant or knowing the kind of plant disease because it does not have experience. In such conditions, a system that can automatically classify plant types or identify types of plant diseases is needed using machine learning (Yamparala et al., 2020) (Ranjan et al., 2015) (Prasetyo, 2016) (Arivazhagan et al., 2013) ) (Rumpf et al., 2010) (Dutta \& Basu, 2013) (Samajpati \& Degadwala, 2016) (Mishra et al., 2021) (Zarrin \& Islam, 2019) (Mia et al., 2020) (Srunitha \& Bharathi, 2018) (Prasad et al., 2016) (Madiwalar \& Wyawahare, 2017) (Kaur et al., 2019) and deep learning (Arya \& Singh, 2019) (Chouhan et al., 2019) (Saldana Ochoa \& Guo, 2019) (Aakif \& Khan, 2015) (Delgado et al., 2019) (Singh et al., 2019) (Nafi'i yah, 2020). Automatic systems for detecting diseases in plants or classification of plant types are beneficial for new farmers and have no experience because they can help work. Classification of plants for ordinary people who are not familiar with crops is a difficult job, and it requires in-depth knowledge of the field from the expert.

Many studies related to the classification of plant species have been carried out using both machine learning and deep learning methods. We mentioned several studies related to plant species classification: classifying mango, papaya, coconut, and banana tree species using the CNN method (Saldana Ochoa \& Guo, 2019); classifying types of mangoes, grapes, oranges, and apples based on images using CNN (Aakif \& Khan, 2015); classifying mango species using machine learning (Ranjan et al., 2015); classifying mango tree species based on geometric features with ANN (Rumpf et al., 2010); classifying plant species using KNN, and Fuzzy Logic (Delgado et al., 2019); classifying types of mangoes with CNN (Zarrin \& Islam, 2019); and classifying tuber types with CNN (Nafi'iyah, 2020).

Previous research shows that the classification of plant species is needed. Because not everyone knows the types of plants, and it takes experts and experts to know the types and characteristics of plants. This study proposes a system for identifying mango plant species based on leaves using the CNN method. The reason for proposing the $\mathrm{CNN}$ method from previous research is that the $\mathrm{CNN}$ method produces good accuracy. Most previous studies to classify plant species use the leaves of the plant. The CNN model proposed refers to research (Saldana Ochoa \& Guo, 2019) (Aakif \& Khan, 2015) (Zarrin \& Islam, 2019) (Nafi'iyah, 2020). The purpose of this study is to propose a CNN architectural model in classifying mango species based on leaf imagery.

\section{RESEARCH METHODS}

\section{Dataset}

Table 1 describes the distribution of the dataset for training and testing. Table 1 describes the types of mangoes divided into 5, namely Alphanso, Amarpali, Ambika, Austin, Kent. The dataset is taken from Kaggle, and only five types of mango are used. Figure 1 is an example of a leaf image.

Table 1. Dataset Sharing

\begin{tabular}{|c|c|c|c|}
\hline No & Type & Training & Test \\
\hline 1 & Alphanso & 24 & 12 \\
\hline 2 & Amarpali & 24 & 12 \\
\hline 3 & Ambika & 24 & 12 \\
\hline 4 & Austin & 24 & 12 \\
\hline 5 & Kent & 24 & 12 \\
\hline
\end{tabular}




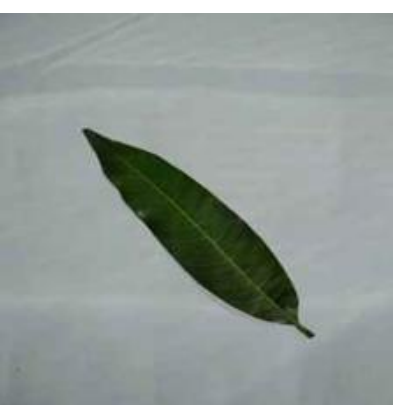

(a)

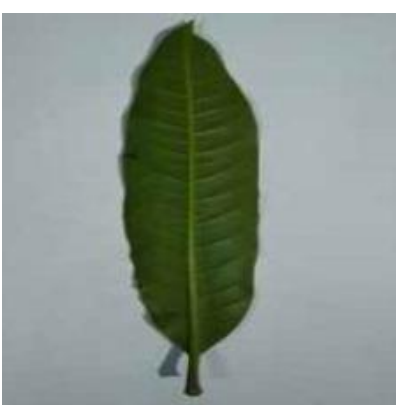

(b)

Figure 1. (a) Manga Type Alphanso (b) Amarpali

The dataset used is an image of colored mango tree leaves with a size of 224 x 224, as shown in Figure 1.

\section{Proposed Research}

This study proposes a CNN architectural model to classify the types of mangoes based on leaf images.

Figure 2 illustrates the flow chart of this research. The $224 \times 224$ color input image is trained based on the CNN architectural model building. We propose $4 \mathrm{CNN}$ models, Figure 2 describes the CNN architecture made with 4 models as shown in Table 2. The difference between the CNN architecture models is the number of convolution layers and the number of nodes in each convolution layer.

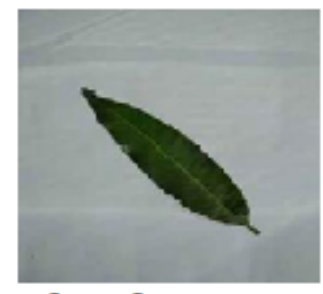

Input Image

$224 \times 224 \times 3$

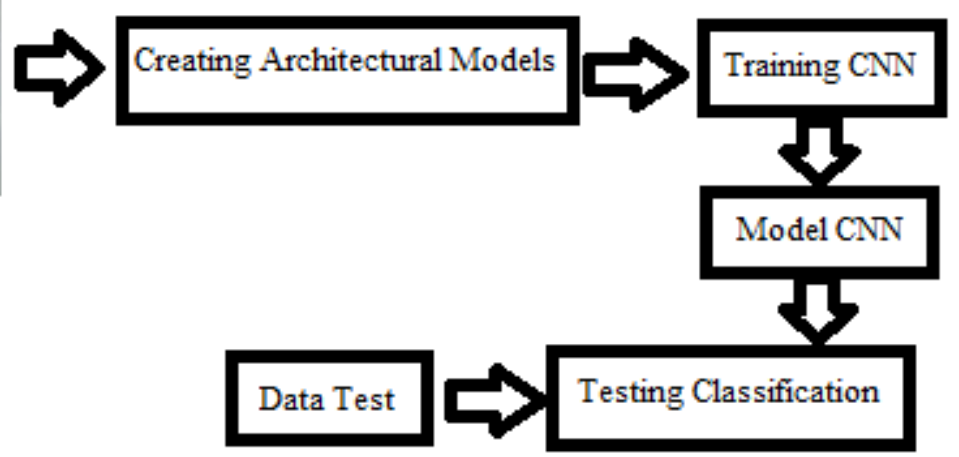

Figure 2. Research Diagram

\section{Convolution Neural Network}

The CNN architectural model proposed in the study is 5; the model is shown in Table 2. Table 2 shows the $\mathrm{CNN}$ model made by explaining the number of parameters.

Table 2. CNN Architecture Model

\begin{tabular}{clr}
\hline No & \multicolumn{1}{c}{ Model Type } & Total Parameters \\
\hline 1 & The First CNN Model & 9700421 \\
2 & Second CNN Model & 7407173 \\
3 & Third CNN Model & 1245989 \\
4 & The Fourth CNN Model & 549797 \\
5 & Transfer Learning InceptionV3 & 21802784 \\
\hline
\end{tabular}




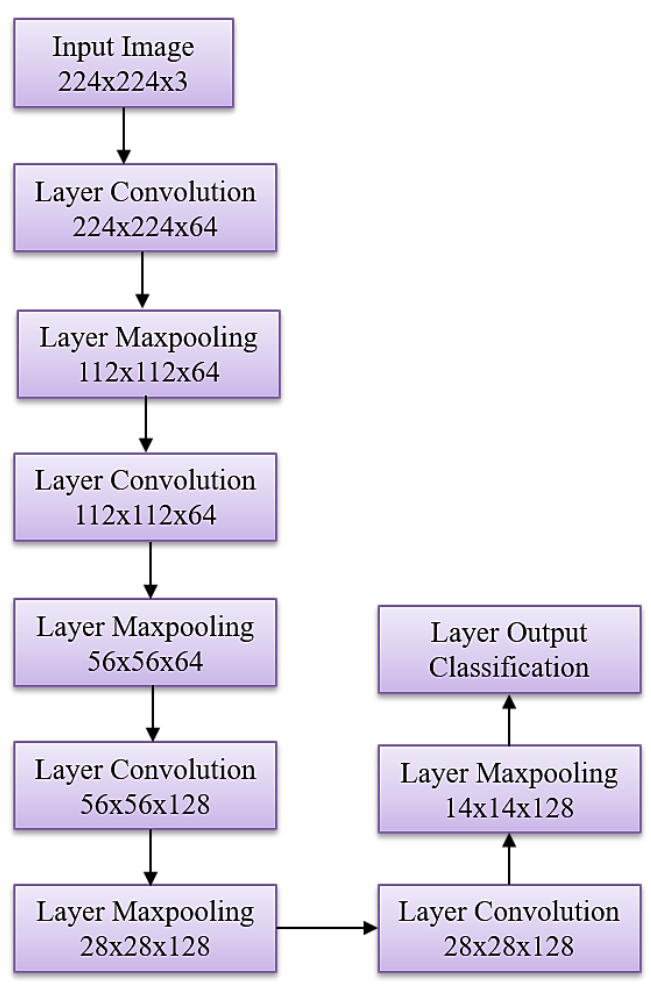

Figure 3. First CNN Architectural Model

Table 2 describes the total parameters of each CNN architectural model. Each architecture has a different number of layers and nodes in each hidden layer. The hidden layer in CNN consists of the convolution layer, the pooling layer, the activation layer, the fully connected layer, and the flatten layer. Each architecture from the first model to the fourth model in Table 2 is described in Figure 3, Figure 4, Figure 5, and Figure 6. Figures 3 and 4 are almost the same as the hidden layers built, only differing in the number of hidden layers. In figure 3 the number there are eight hidden layers, while in Figure 4, there are 10. Simultaneously, the number of nodes in each Convolution and Maxpooling layer is almost the same.

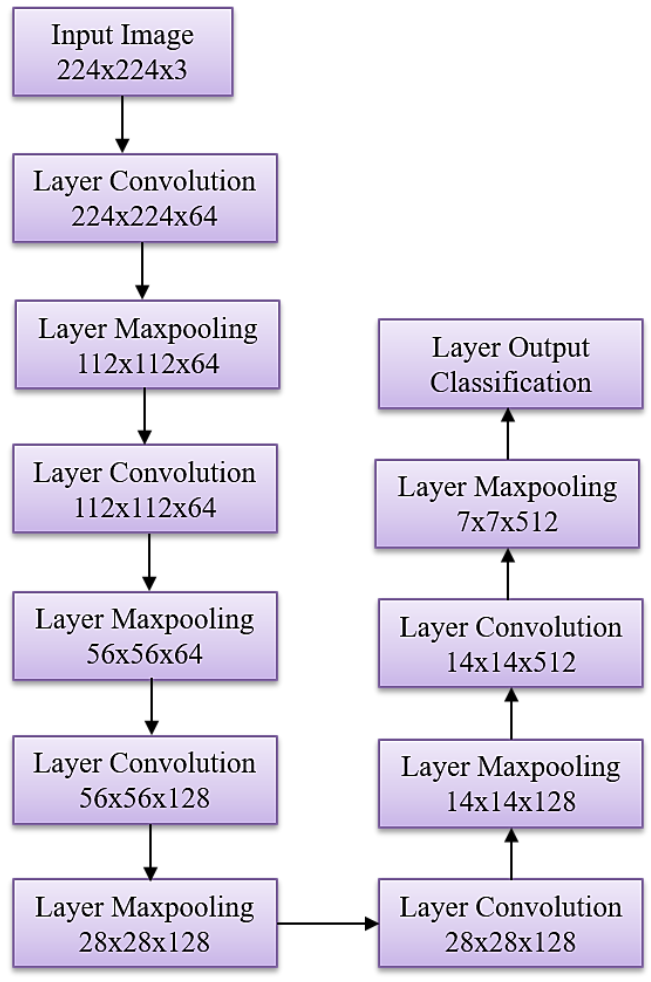


Figure 4. Second CNN Architectural Model

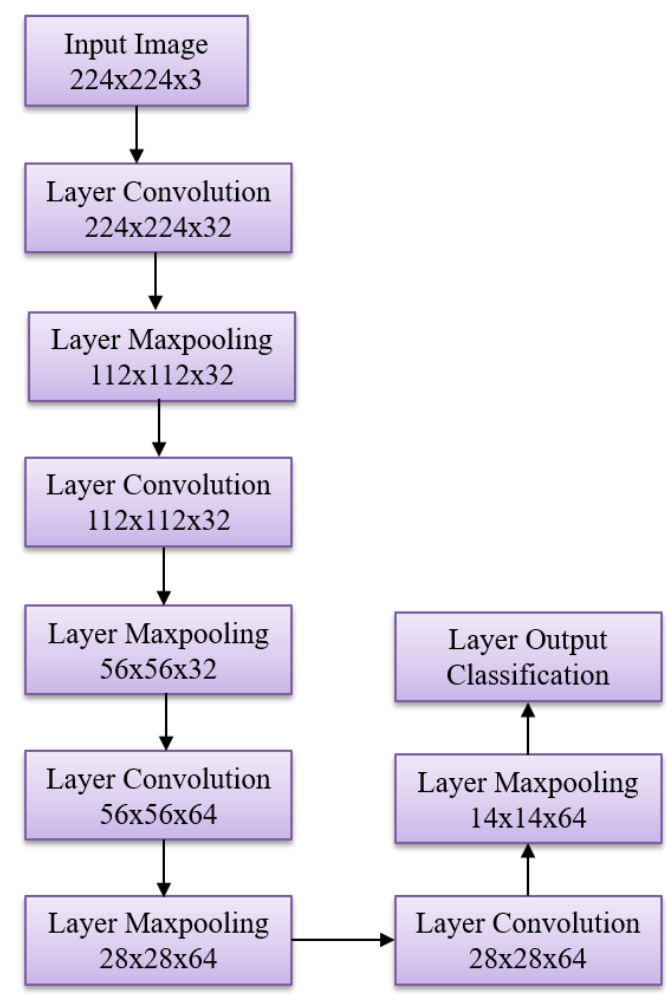

Figure 5. Third CNN Architectural Model

The explanation from Figure 3 to Figure 6 shows the CNN architecture built. The difference between each architecture is the number of convolution layers and max-pooling layer and the number of nodes in each convolution layer and max-pooling layer.

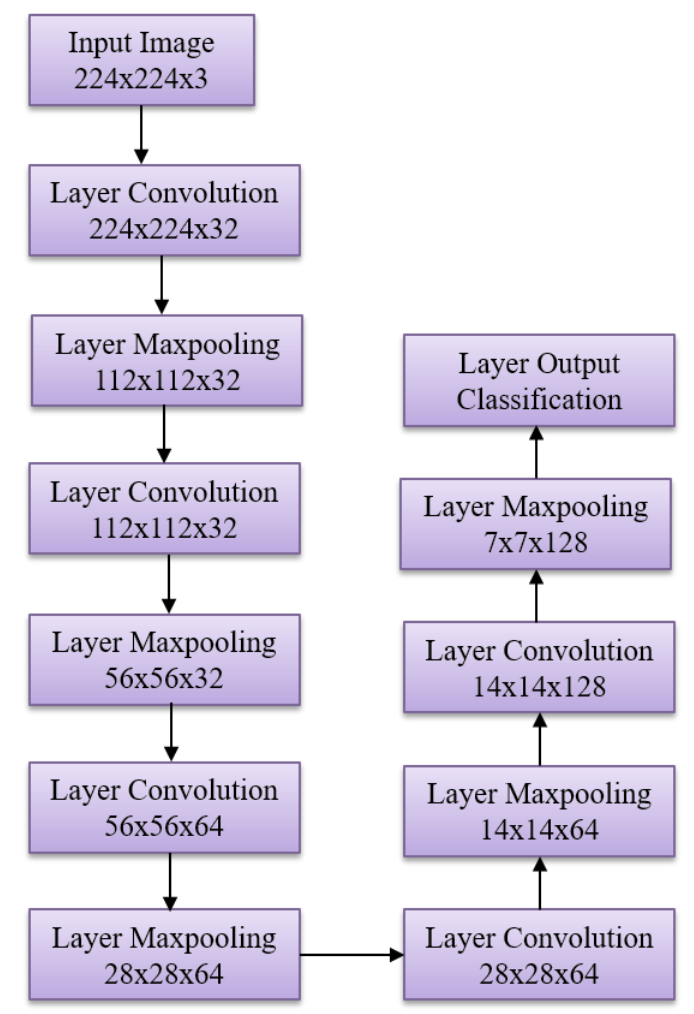

Figure 6. Fourth CNN Architectural Model 


\section{RESULTS AND DISCUSSION}

This study proposes $4 \mathrm{CNN}$ architectural models and uses InceptionV3 transfer learning. Each architectural model differs in the number of layers and nodes in the convolution layer, the max-pooling layer. The number of parameters of each $\mathrm{CNN}$ architectural model is different. The most significant number of parameters in the InceptionV3 learning transfer model. Simultaneously, the most considerable number of proposed model parameters is the first model in Figure 3. Each proposed architectural model is trained and evaluated, as many as 50 epochs of training are carried out. We understand that the number of datasets used is minimal, namely 24 of each type of mango shown in Table 1, so we also augment the training. When we did our training, we used error testing with categorical loss cross-entropy. Equation 1 describes the formula for calculating the error value. The error value can also be called the loss value, namely the loss value in training or evaluation is the value of the difference in calculations between the prediction results symbolized in $\hat{y}_{i}$ the actual data or the fact data symbolized in $y_{i}$. The greater the value of the difference between the predicted value and the actual value, the less good the model. The greater the accuracy value of a model, the better the model. The accuracy value is the same value between the actual value $y_{i}$ and the predicted value $\hat{y}_{i}$. The loss and accuracy values of the four CNN architectural models that have been experimented are in Table 3.

$$
\text { Loss }=\frac{1}{N} \sum_{i=1}^{N}-\left(y_{i} \log \hat{y}_{i}+\left(\left(1-y_{i}\right) \log \left(1-\hat{y}_{i}\right)\right)\right)
$$

Table 3. Accuracy and Error Results from Training and Evaluation

\begin{tabular}{rlrrrr}
\hline & & \multicolumn{2}{c}{ Training } & \multicolumn{2}{c}{ Evaluation } \\
\cline { 3 - 6 } No & \multicolumn{1}{c}{ Type Model CNN } & Loss & Accuracy & Loss & Accuracy \\
\hline 1 & The First CNN Model & 1.396 & 0.35 & 1.26 & 0.48 \\
2 & Second CNN Model & 1.4015 & 0.375 & 1.46 & 0.4 \\
3 & Third CNN Model & 1.33 & 0.43 & 1.23 & 0.55 \\
4 & The Fourth CNN Model & 1.446 & 0.325 & 1.431 & 0.367 \\
5 & Transfer Learning InceptionV3 & 1.61 & 0.2 & 1.61 & 0.2 \\
\hline
\end{tabular}

The purpose of calculating the error value for each iteration in training is to improve each node's weight value in the convolution and max-pooling layers. The explanation of Equation 1 is that $\mathrm{y}$ is the actual data, while the algorithm's output data $\hat{y}$. Table 3 describes the error value and accuracy of the training and evaluation process of each of the proposed CNN model architectures and transfer learning. Each training and evaluation process of the CNN architectural model is shown in Figure 7 to Figure 11. Figure 7 describes the performance of the loss value and the accuracy of the first CNN architectural model. Each Figure 7 to 11, the left represents the performance accuracy, and the right part explains the value of the loss. 

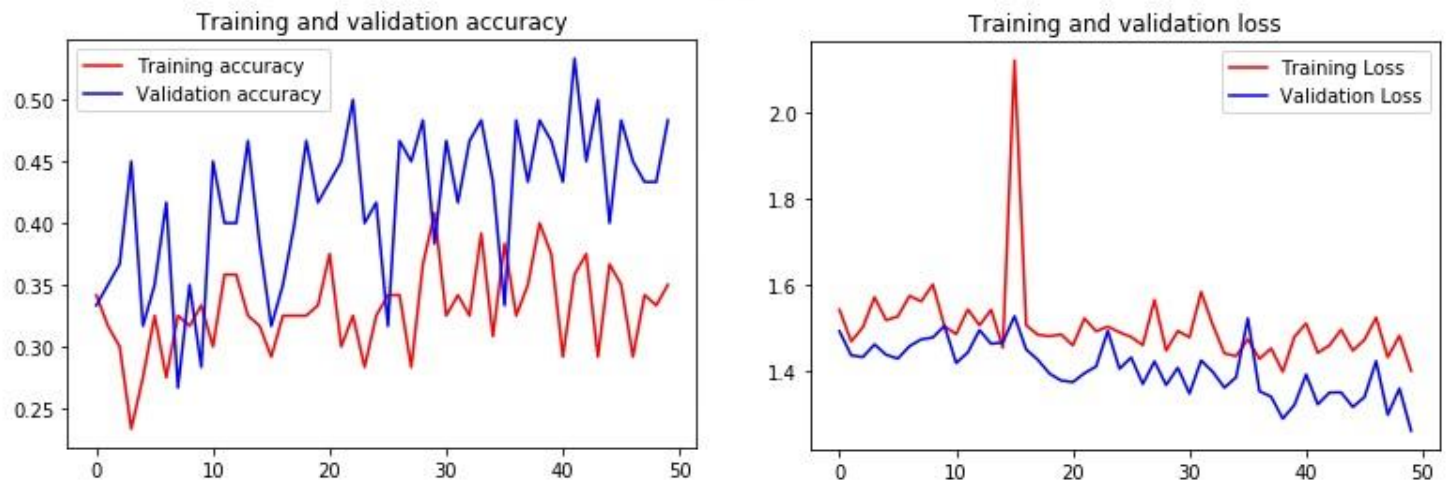

Figure 7. First CNN Model Loss Value and Accuracy Graph
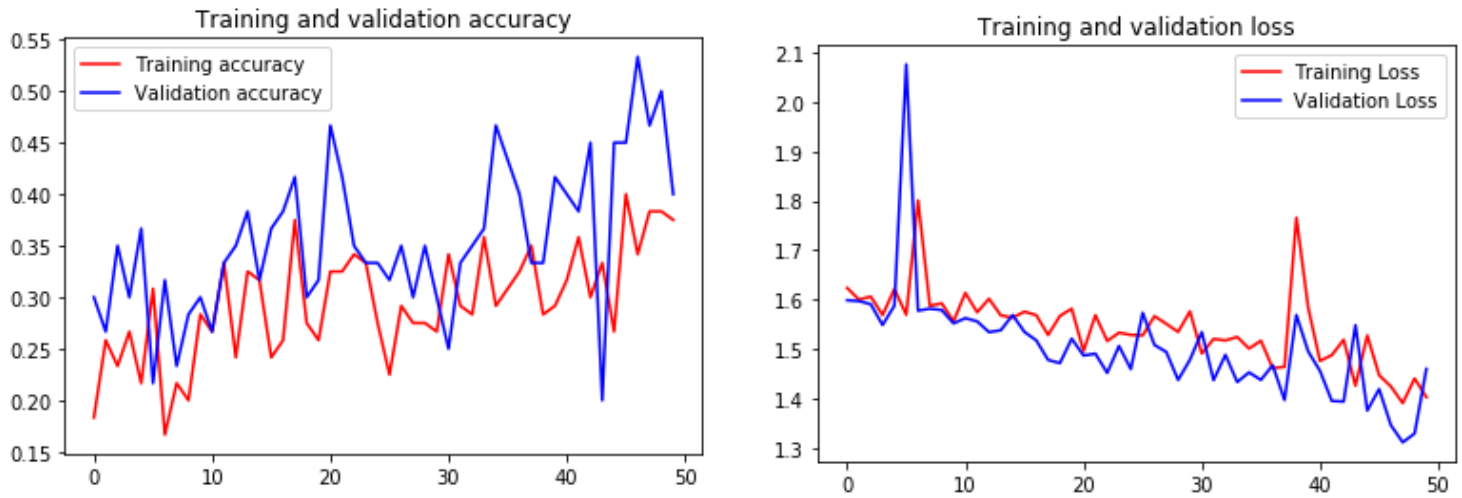

Figure 8. Graph of Second CNN Model Loss and Accuracy
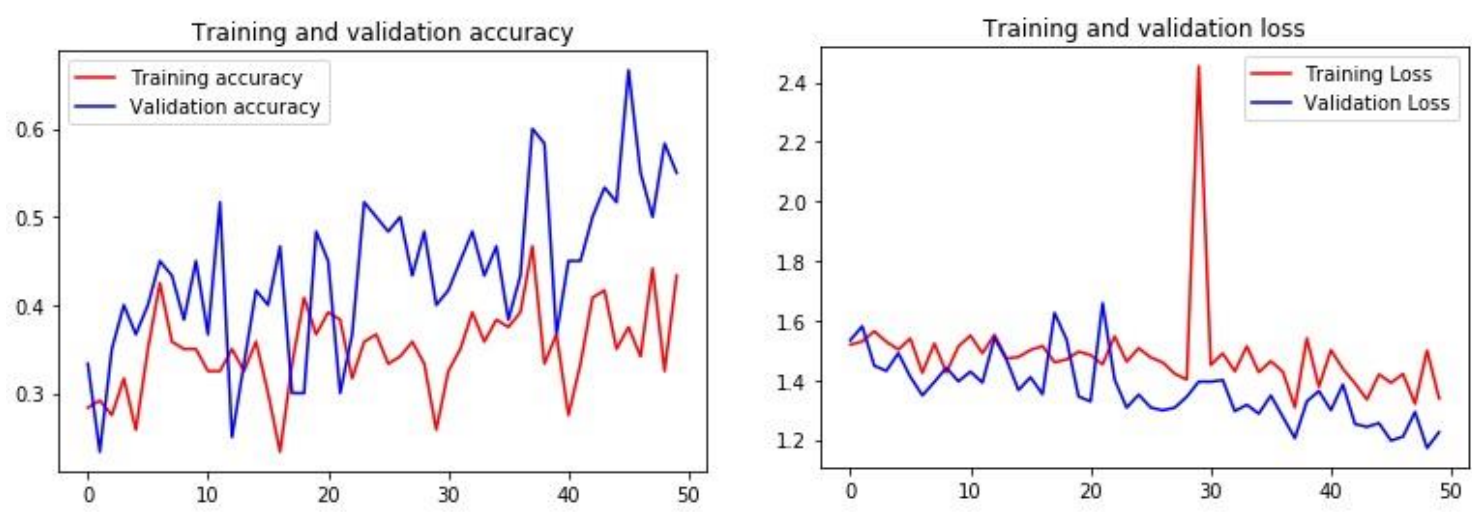

Figure 9. Graph of Third CNN Model Loss and Accuracy
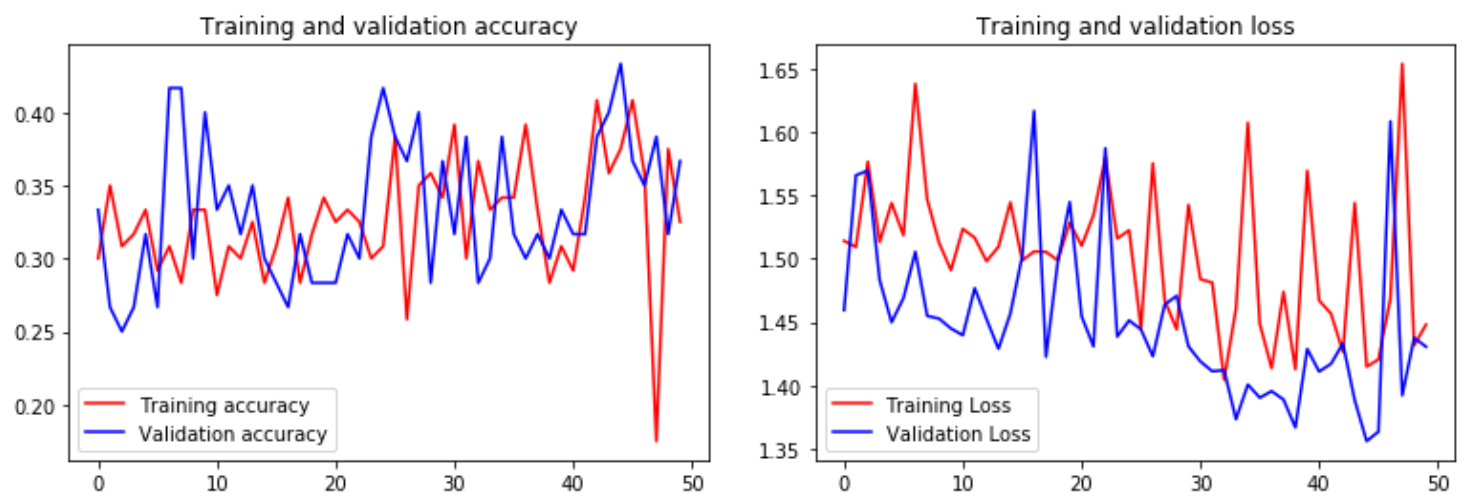

Figure 10. Graph of the Value Loss and Accuracy of the Fourth CNN Model 

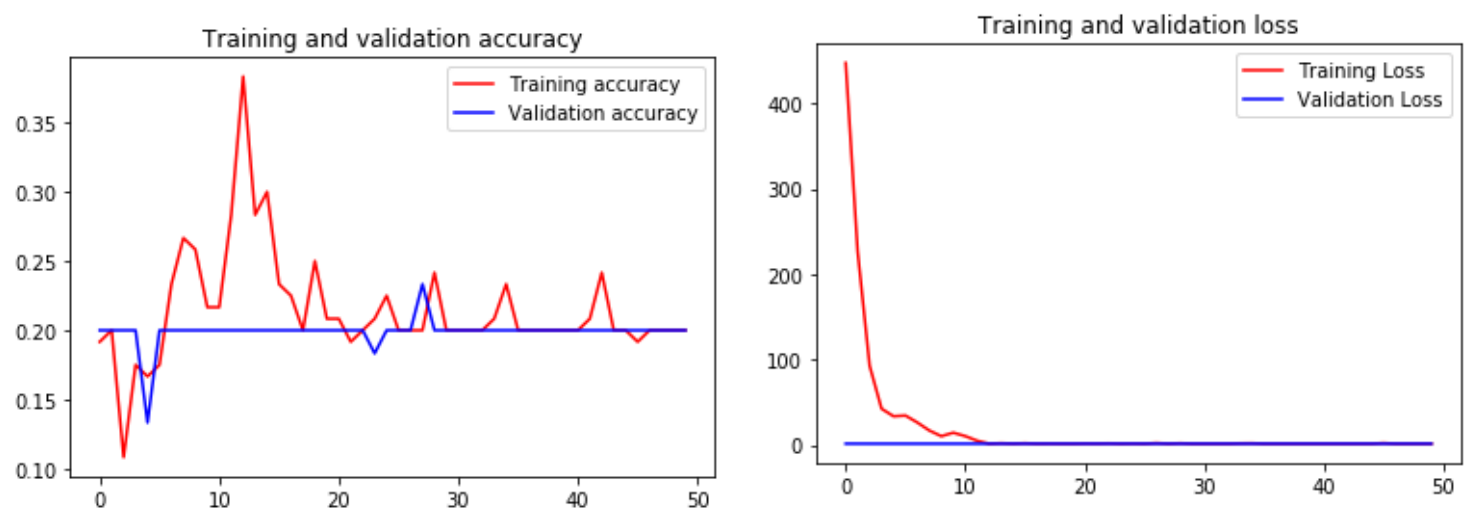

Figure 11. Graph of Value Loss and Accuracy of CNN Transfer Learning Model

Figure 8 describes the performance of the loss values and the accuracy of the second CNN architectural model. Figure 9 represents the performance of the loss value and the accuracy of the third $\mathrm{CNN}$ architectural model. Figure 10 illustrates the implementation of the loss value and the accuracy of the fourth CNN architectural model. Figure 11 describes the performance of the loss value and accuracy of the CNN InceptionV3 transfer learning model. Based on Table 3, the proposed model has the lowest loss value compared to CNN transfer learning. The proposed model has $4 \mathrm{CNN}$ architectures, and the third model has the best performance, with an evaluation loss value of 1.431 and an accuracy of 0.55 . The highest accuracy is the third CNN architectural model with the number of parameters 1245989.

\section{CONCLUSIONS AND RECOMMENDATIONS}

Based on the evaluation test results of the proposed CNN architectural model, the best architectural model is the third. The number of third CNN architecture parameters is 1245989 , with the loss and accuracy values when evaluating is 1.431 and 0.55 . The parameter number is learning transfer InceptionV3 21,802,784, but the transfer of learning shows the lowest accuracy and highest lossy, namely, 0.2 and 1.61. It can conclude that the third architectural model has the best performance compared to the other four models.

\section{ACKNOWLEDGEMENT}

Thank you to all those who helped correct and publish this article, especially to the Lamongan Islamic University.

\section{REFERENCES/BIBLIOGRAPHY}

Aakif, A., \& Khan, M. F. (2015). Automatic classification of plants based on their leaves. Biosystems Engineering, 139. https://doi.org/10.1016/j.biosystemseng.2015.08.003

Arivazhagan, S., Shebiah, R. N., Ananthi, S., \& Vishnu Varthini, S. (2013). Detection of unhealthy region of plant leaves and classification of plant leaf diseases using texture features. Agricultural Engineering International: CIGR Journal, 15(1).

Arya, S., \& Singh, R. (2019). A Comparative Study of CNN and AlexNet for Detection of Disease in Potato and Mango leaf. IEEE International Conference on Issues and Challenges in Intelligent Computing Techniques, ICICT 2019. https://doi.org/10.1109/ICICT46931.2019.8977648

Chouhan, S. S., Kaul, A., \& Singh, U. P. (2019). A deep learning approach for the classification of diseased plant leaf images. Proceedings of the 4th International Conference on Communication and Electronics Systems, ICCES 2019. https://doi.org/10.1109/ICCES45898.2019.9002201 
Delgado, N. O., Arboleda, E. R., Dioses, J. L., \& Dellosa, R. M. (2019). Identification of mango leaves using artificial intelligence. International Journal of Scientific and Technology Research, 8(12), 2864-2868.

Dutta, L., \& Basu, T. K. (2013). Extraction And Optimization Of Leaves Images Of Mango Trees And Classification Using Ann. International Journal of Recent Advances in Engineering \& Technology (IJRAET) ISSN (Online) 2347-2812, 1(3), 46-51.

Kaur, S., Pandey, S., \& Goel, S. (2019). Plants Disease Identification and Classification Through Leaf Images: A Survey. Archives of Computational Methods in Engineering, 26(2). https://doi.org/10.1007/s11831-018-9255-6

Madiwalar, S. C., \& Wyawahare, M. V. (2017). Plant disease identification: A comparative study. 2017 International Conference on Data Management, Analytics and Innovation, ICDMAI 2017. https://doi.org/10.1109/ICDMAI.2017.8073478

Mia, M. R., Roy, S., Das, S. K., \& Rahman, M. A. (2020). Mango leaf disease recognition using neural network and support vector machine. Iran Journal of Computer Science, 3(3). https://doi.org/10.1007/s42044-020-00057-z

Mishra, S., Ellappan, V., Satapathy, S., Dengia, G., Mulatu, B. T., \& Tadele, F. (2021). Identification and classification of mango leaf disease using wavelet transform based segmentation and wavelet neural network model. Annals of the Romanian Society for Cell Biology, 25(2).

Nafi'iyah, N. (2020). Tuber Type Classification Based on Image of Bulbs with Deep Learning. 7th International Conference on ICT for Smart Society: AIoT for Smart Society, ICISS 2020 - Proceeding. https://doi.org/10.1109/ICISS50791.2020.9307584

Prasad, S., Peddoju, S. K., \& Ghosh, D. (2016). Multi-resolution mobile vision system for plant leaf disease diagnosis. Signal, Image and Video Processing, 10(2). https://doi.org/10.1007/s11760-015-0751-y

Prasetyo, E. (2016). Detection of mango tree varieties based on image processing. Indonesian Journal of Science and Technology, 1(2). https://doi.org/10.17509/ijost.v1i2.3800

Ranjan, M., Weginwar, M. R., Joshi, N., \& Ingole, P. A. B. (2015). Detection and Classification of Leaf Disease using Artificial Neural Network. International Journal of Technical Research and Application(IJTRA), 3(3).

Razi, F. A. (2012). An Analysis of COVID-19 using X-ray Image Segmentation based Graph Cut and Box Counting Fractal Dimension. Telematika, 14(1). https://doi.org/10.35671/telematika.v14i1.1217

Rumpf, T., Mahlein, A. K., Steiner, U., Oerke, E. C., Dehne, H. W., \& Plümer, L. (2010). Early detection and classification of plant diseases with Support Vector Machines based on hyperspectral reflectance. Computers and Electronics in Agriculture, 74(1). https://doi.org/10.1016/j.compag.2010.06.009

Saldana Ochoa, K., \& Guo, Z. (2019). A framework for the management of agricultural resources with automated aerial imagery detection. Computers and Electronics in Agriculture, 162. https://doi.org/10.1016/j.compag.2019.03.028

Samajpati, B. J., \& Degadwala, S. D. (2016). Hybrid approach for apple fruit diseases detection and classification using random forest classifier. International Conference on Communication and Signal Processing, ICCSP 2016. https://doi.org/10.1109/ICCSP.2016.7754302

Singh, U. P., Chouhan, S. S., Jain, S., \& Jain, S. (2019). Multilayer Convolution Neural Network for the Classification of Mango Leaves Infected by Anthracnose Disease. IEEE Access, 7. https://doi.org/10.1109/ACCESS.2019.2907383

Srunitha, K., \& Bharathi, D. (2018). Mango leaf unhealthy region detection and classification. In Lecture Notes in Computational Vision and Biomechanics (Vol. 28). https://doi.org/10.1007/978-3-31971767-8_35 
Yamparala, R., Challa, R., Kantharao, V., \& Krishna, P. S. R. (2020). Computerized classification of fruits using convolution neural network. 2020 7th International Conference on Smart Structures and Systems, ICSSS 2020. https://doi.org/10.1109/ICSSS49621.2020.9202305

Zarrin, I., \& Islam, S. (2019). Leaf Based Trees Identification Using Convolutional Neural Network. 2019 IEEE 5th International Conference for Convergence in Technology, I2CT 2019. https://doi.org/10.1109/I2CT45611.2019.9033914 